\title{
S-Nitrosoglutathione inhibits platelet activation and deposition in coronary artery saphenous vein grafts in vitro and in vivo
}

\author{
E Salas, E J Langford, M T Marrinan, J F Martin, S Moncada, A J de Belder
}

\begin{abstract}
Objective-To investigate platelet activation and deposition in human saphenous vein and internal mammary artery grafts following coronary artery bypass in vitro and in vivo, as well as inhibition of activation by the platelet selective nitric oxide donor S-nitrosoglutathione (GSNO).

Design-Controlled in vitro and in vivo studies.

Setting-Tertiary cardiac centre.

Patients-24 patients undergoing coronary artery bypass surgery requiring vein and artery grafts.

Interventions-In vitro: human platelet rich plasma was perfused through segments of vein and artery, with or without GSNO $10^{-6} \mathrm{M}$, and the platelet count was measured in the effluent. In vivo: indium111 labelled antibody against the platelet $\alpha$ granule protein GMP-140 was injected at the end of coronary bypass grafting and $\gamma$ counts were compared between vein and artery grafts with or without systemic infusion of GSNO (40 $\mathrm{nmol} / \mathrm{min})$.
\end{abstract}

Results-In vitro: platelet count in perfused vein ( $<70 \%$ of baseline) decreased more than in artery segments $(89-94 \%$ of baseline) $(p<0.001)$. The platelet count was unchanged with GSNO in vein and artery segments. In vivo: $\gamma$ counts were greater at all time points over vein than artery grafts $(p<0.05)$, and were reduced by infusion of GSNO (p < 0.05).

Conclusions-Platelet activation is greater in vein than in artery grafts in vitro and in vivo. Activation, which contributes to early vein graft failure, was inhibited by GSNO.

(Heart 1998;80:146-150)

Keywords: coronary artery bypass surgery; platelet activation; S-nitrosoglutathione; ischaemic heart disease

Cardiothoracic

Surgery, King's

College Hospital

M T Marrinan

Cruciform Project, University College,

London, UK

J F Martin

S Moncada

Correspondence to: Dr A J de Belder,

Department of Cardiology,

King's College Hospital,

Denmark Hill, London

SE5 9RS, UK.

Accepted for publication 4 March 1998

Coronary artery bypass surgery is a successful treatment for ischaemic heart disease. Long term outcome, however, depends on the patency of coronary bypass conduits ${ }^{1}$ and this remains disappointing, with recurrence of anginal symptoms in up to $22 \%$ of patients at two years. ${ }^{2}$ The situation has improved with the use of internal mammary artery bypass conduits, which have a $90 \%$ patency at 10 years. $^{3}$ Saphenous vein grafts, which are the main choice for this operation, however, have a $15-30 \%$ occlusion rate within the first year after surgery, with half occluding in the first few weeks. ${ }^{4}$ Activated platelets have an important role in the pathogenesis of vein graft failure, initially as a trigger to thrombosis and subsequently leading to intimal hyperplasia. ${ }^{4} \mathrm{An}$ tiplatelet treatment with aspirin reduces occlusion rates in the first year but does not achieve patency rates comparable with those of arterial grafts. $^{5}$

The L-arginine nitric oxide (NO) pathway is an important regulator of platelet vessel wall interactions and vascular tone. Endothelium derived NO inhibits platelet aggregation ${ }^{6}$ and adhesion, ${ }^{7}$ and the reduced ability of vein grafts compared with arterial conduits to generate NO may account for the poor clinical outcome of vein grafts. ${ }^{89}$ Therefore, NO donors would be a logical therapeutic strategy to supplement the grafted vein during and after surgery. Delivery of NO to patients can be achieved using organic nitrates, such as glyceryl trinitrate, but the antiplatelet effect of these compounds is limited by their concomitant hypotensive effect. ${ }^{10}$ We recently reported that the stable nitrosothiol S-nitrosoglutathione (GSNO) has significant antiplatelet effects at doses that do not alter vascular tone significantly in man. ${ }^{11-13}$

This study aimed to: measure platelet deposition within vein and arterial grafts in vitro; determine the extent of platelet activation in saphenous vein and internal mammary artery grafts in vivo following coronary artery bypass surgery; and to investigate the effect of GSNO on these platelet activities.

Patients and methods

The study was approved by the King's Healthcare Trust ethics committee. In addition, the study received approval from the ARSAC for radioisotope administration for clinical investigation. Patients were provided with an information sheet and fully informed consent was obtained the day before surgery.

The criterion for study entry was the construction of both internal mammary artery and saphenous vein bypass conduits in patients undergoing elective coronary artery bypass surgery for stable angina. Aspirin was discontinued seven to 10 days before surgery. Other than their usual antianginal medication, patients did not receive drugs with antiplatelet effects. Patients took their usual medication on the morning of surgery (table 1). Twenty four patients were enrolled in the study, divided equally into two groups - the in vitro or in vivo study. 
Table 1 Details of the two groups of patients

\begin{tabular}{lll}
\hline & $\begin{array}{l}\text { In vivo study } \\
(n=12)\end{array}$ & $\begin{array}{l}\text { In vitro study } \\
(n=12)\end{array}$ \\
\hline Mean (SD) age (years) & $64(9.4)$ & $62(11.2)$ \\
Sex $(\mathrm{M} / \mathrm{F})$ & $9 / 3$ & $8 / 4$ \\
Nitrates & 12 & 12 \\
$\beta$ Blockers & 9 & 8 \\
Calcium antagonists & 10 & 10 \\
\hline
\end{tabular}

All patients took their drugs on the morning of the operation.

IN VITRO STUDY

Distal segments of saphenous vein and internal mammary artery were obtained from 12 patients (table 1). The segments were placed in normal saline, excess connective tissue was carefully removed, and rings $(1.5 \mathrm{~cm})$ were mounted on a double walled chamber at $37^{\circ} \mathrm{C}$. Venous blood $(27 \mathrm{ml})$ was taken from a large central venous catheter into a syringe containing $3 \mathrm{ml} 3.15 \%$ trisodium citrate before starting cardiopulmonary bypass. Platelet rich plasma (PRP) was obtained by centrifugation $(220 \times g)$ at $22^{\circ} \mathrm{C}$ for 20 minutes. Autologous PRP was infused through the vascular segments using a 21 gauge butterfly needle, with a Harvard " 11 " syringe pump at a flow rate of $20 \mathrm{ml} /$ hour. Samples from six patients acted as controls, while GSNO $\left(10^{-6} \mathrm{M}\right)$ was added to the PRP of the other six. Samples of PRP were collected after passing through the vascular segment at five, 10, 15, and 20 minutes, each sample being collected over a period of one minute. Baseline collections were taken at the beginning and end of each experiment, collecting PRP that was not infused through the vascular segment for one minute. PRP was infused through both artery and vein segments at random for each patient. Platelet counts were measured for all PRP samples using a Coulter T-540 platelet counter (Coulter Electronics, Hialeah, Florida, USA).

IN VIVO STUDY

Platelet activation marker labelling protocol

Expression of platelet activation antigens may be measured using labelled monoclonal antibodies. The platelet $\alpha$ granule protein GMP140 (PADGEM, P selectin) is released onto the platelet surface only after platelet activation, so radiolabelled antibody to GMP-140 can be used as a marker for activated platelets with high in vivo specificity. Its use in humans is safe. ${ }^{14}$ Labelling of this antibody was performed as previously described, ${ }^{15}{ }^{16}$ with some minor modifications. Briefly, indium-111 $(50 \mu \mathrm{l})$ was transferred to an Eppendorf tube to which $37.5 \mu \mathrm{l}\left(0.75 \times\right.$ volume $\left.{ }^{111} \mathrm{In}\right) \quad 0.1 \mathrm{M}$ hydrochloric acid was added, followed immedi-

Table 2 Platelet counts following infusion through saphenous vein and internal mammary artery segments in vitro

\begin{tabular}{llllll}
\hline & \multicolumn{3}{l}{ Vein } & & \multicolumn{2}{l}{ Artery } \\
\cline { 2 - 3 } \cline { 5 - 6 } Time & No GSNO & With GSNO & & No GNSO & With GSNO \\
\hline 5 & $70.0(0.5)$ & $97.7(1.6)$ & & $93.9(3.5)$ & $99(2.1)$ \\
10 & $68.0(1.6)$ & $96.1(2.0)$ & & $94.0(2.5)$ & $97(1.3)$ \\
15 & $67.0(2.0)$ & $97.7(1.6)$ & & $94.0(4.0)$ & $99.5(1.9)$ \\
20 & $67.0(2.0)$ & $96.7(1.5)$ & & $89.0(3.0)$ & $99.5(2.1)$ \\
\hline
\end{tabular}

Counts are expressed as percentage of baseline count.

Time points are time in minutes after starting infusion.

Six patients in each group. ately by $25 \mu \mathrm{l}$ phosphate buffer $(0.14 \mathrm{M}$, $\mathrm{pH} 7.4)\left(0.5 \times\right.$ volume $\left.{ }^{111} \mathrm{In}\right)$. Anti GMP-140 antibody $(5 \mu \mathrm{l})$ containing $19 \mu \mathrm{g} / \mu \mathrm{l}$ was added and the tube placed in an ultrasonic bath for five minutes. A stock solution of Chloramine-T at $10 \mu \mathrm{g} / \mu \mathrm{l}$ was prepared with sterile saline during sonification. The tube was removed from the bath after five minutes, Chloramine-T solution $(32 \mu \mathrm{l})$ was added, and the tube replaced in the ultrasonic bath for three minutes. During this incubation a stock solution of $10 \mu \mathrm{g} / \mu \mathrm{l}$ sodium metabisulphite was prepared in sterile saline. This solution $(32 \mu \mathrm{l})$ was added after three minutes. The final solution was administered as a bolus injection in the in vivo investigation.

All patients were anticoagulated during coronary artery bypass surgery with heparin, to an activated clotting time of greater than 400 seconds, and cooled to $26^{\circ} \mathrm{C}$ during bypass using a membrane oxygenator at comparable flow rates. During the operation, and throughout the study, glyceryl trinitrate was given as a vasodilator, the dose being titrated against the patient's blood pressure as is routine practice in our institution. The study started when bypass surgery was complete, and the patient was successfully weaned off cardiopulmonary bypass, but before closure of the sternum. A bolus of ${ }^{111}$ In labelled antiGMP-140 monoclonal antibody (Immunotech, Birmingham, UK) $(260 \mu \mathrm{Ci}, 100 \mu \mathrm{g})$ was injected through a central venous catheter in 12 patients. GSNO $(40 \mathrm{nmol} / \mathrm{min}$; the dose being calculated by extrapolation from our original studies in the human forearm ${ }^{11}$ ) was continuously infused throughout the study in six patients, starting immediately after injection of radiolabelled antibody. The other patients acted as controls. Heart rate and mean arterial pressure were recorded continuously throughout infusion. $\gamma$ Emission was recorded using a Teledyne isotope detector attached to an Acemate amplifier (EG \& G Instruments, Wokingham, Berks, UK) five minutes after antibody injection. The probes were localised over the vein and artery bypass conduits under sterile conditions by the surgeon, using the aorta as a reference standard. The data were stored on disk and analysed using the Automated Isotope Monitoring System (Mumed, UK). As absolute activated platelet numbers cannot be determined from this protocol, the radioactivity counts over the study vessel conduit, in the presence or absence of GSNO, is expressed as a percentage of counts over the aorta.

\section{STATISTICAL ANALYSIS}

Values for in vitro and in vivo studies are expressed as mean (SEM). In both studies, values at each time point are compared with baseline using analysis of variance (ANOVA). Comparison between artery and vein grafts and between controls and GSNO treatment are made using ANOVA; $\mathrm{p}<0.05$ is considered significant. 

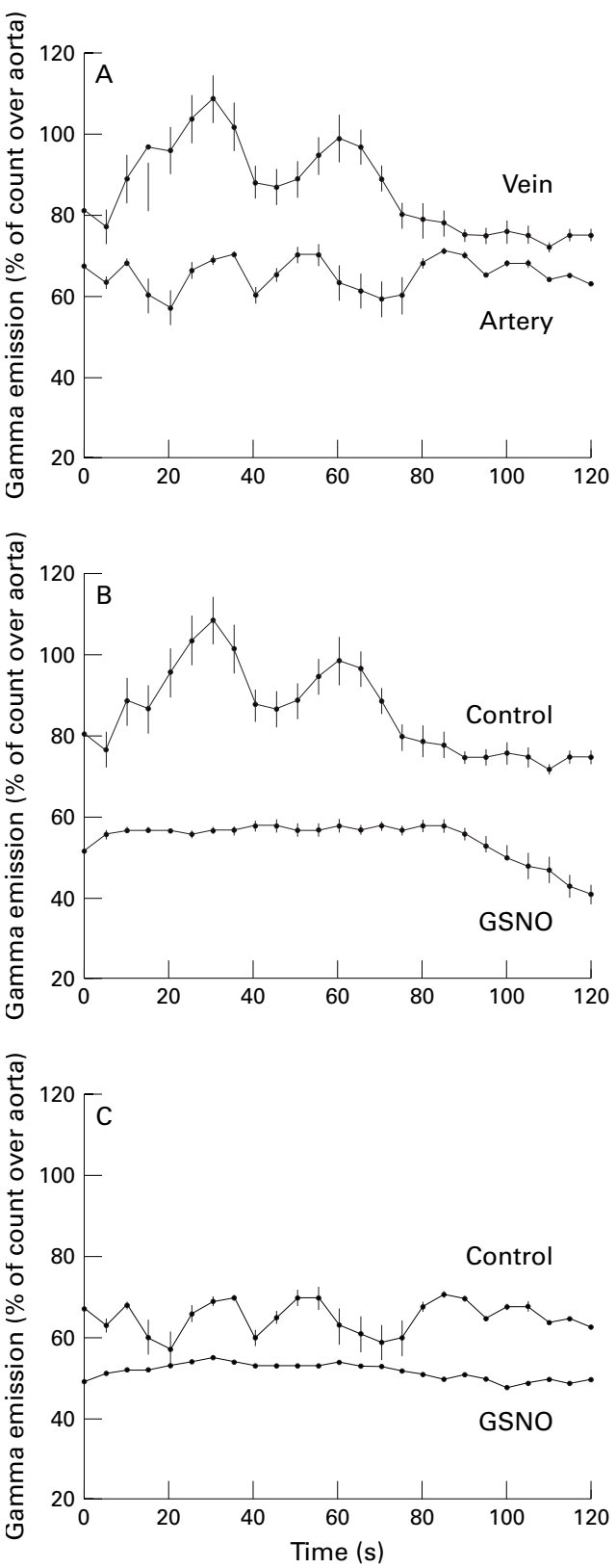

Figure 1 In vivo platelet GMP-140 expression detected with a $\gamma$ camera in saphenous vein and internal mammary artery grafts after coronary artery bypass surgery. Counts are expressed as a percentage of counts detected in the aorta. Each point represents the mean (SEM) of six patients. (A) Platelet activation is greater in vein grafts than in artery grafts. (B) Counts over vein grafts in the presence (GSNO) or absence (control) of GSNO (40 nmol/min).

(C) Counts over artery grafts in the presence (GSNO) or absence (control) of GSNO (40 nmol/min). Platelet activation in vein and arterial grafts is inhibited by GSNO.

Results

Patients were well matched for age, sex, smoking habits, and medication (table 1). The dose of glyceryl trinitrate in the in vivo study was the same as in the GSNO treated and control groups.

IN VITRO STUDY

PRP infused through vascular segments in the six control patients caused the platelet count to decrease more after perfusion through the saphenous vein graft segments than through the internal mammary artery segments (table 2 ). The platelet count in PRP infused through the saphenous vein segments (expressed as a percentage of the baseline platelet count in PRP not infused through the vascular segments) decreased to $70(0.5) \%$ at five minutes after starting infusion, with similar decreases at 10,15 , and 20 minutes $(p<0.001$ at each time point compared with baseline). Thus the consistent decrease in platelet count in the vein segments did not vary with duration of the infusion. In contrast, the platelet count in PRP infused through the internal mammary artery segments decreased to a smaller extent, to 93.9 (3.5)\% at five minutes after starting infusion, with similar values after 10 and 15 minutes, and a slightly greater decrease, to 89 (3)\%, at 20 minutes. This reduction was significant compared with baseline only at the 20 minute time point $(p=0.02)$. There was no difference between baseline platelet counts at the start and end of each study, indicating no change with time in the platelet count of infused PRP. When GSNO $\left(10^{-6} \mathrm{M}\right)$ was infused concurrently with PRP, there was no significant decrease in platelet count at any time points following infusion through either saphenous vein or internal mammary artery segments (table 2).

IN VIVO STUDY

GMP-140 expression, as measured by $\gamma$ counting, was significantly greater in saphenous vein grafts than in the internal mammary artery conduits (fig $1 \mathrm{~A})(\mathrm{p}<0.05)$. GMP-140 expression in vein and arterial grafts in patients who received GSNO $(40 \mathrm{nmol} / \mathrm{min})$ was lower than in grafts in the control group $(\mathrm{p}<0.05)$ (figs $1 \mathrm{~B}$ and $1 \mathrm{C}$ ). Platelet activation in vein grafts of the group treated with GSNO was similar to, or lower than, that in arterial grafts of the control group, but higher than that in arterial grafts treated with GSNO. GSNO infusion did not cause a significant change in mean arterial pressure or heart rate.

\section{Discussion}

There are many theoretical reasons why platelet activation may have a role in the pathogenesis of early and late failure of coronary bypass vein grafts, but direct evidence of in vivo activation has been lacking. In the in vivo study increased labelling of GMP-140 within saphenous vein grafts compared with that in internal mammary artery grafts immediately after coronary bypass surgery suggests increased numbers of activated platelets secreting granule contents. This finding may arise from increased local activation of platelets within the vein grafts or to deposition of activated platelets and provides evidence for a difference in local platelet activation and granule secretion between artery and vein grafts. In addition, the in vitro experiments showed a greater decrease in platelet count following infusion through saphenous vein than in internal mammary artery segments.

The decrease in platelet count following infusion through the vein segments may be 
caused by deposition on the vein endothelium or platelet aggregation, or both. Histological study or electron microscopy may have enabled this question to be answered, but the aim of this study was solely to determine whether a difference exists between the different conduits and whether this may be influenced by GSNO, so these techniques were not used. This process was constant throughout the time points studied, so it is unlikely to be simply deposition of a platelet monolayer. The smaller decrease in platelet count in internal mammary artery segments suggests that arterial endothelium is a less adhesive surface for platelets. While this study shows a difference between artery and vein segments under these conditions, reflecting the changes seen in vivo, the experimental conditions are very different in terms of flow rate and pressure, affecting distension of the vascular segments and contact time between platelets and the vascular wall. The in vitro findings are therefore unlikely to give a quantitative indication of in vivo platelet activation.

The underlying mechanisms may relate to differences in the endothelial responses of arterial and vein grafts as endothelium dependent relaxation is reduced in vein grafts compared with that in arterial grafts in vitro. ${ }^{17} \mathrm{NO}$ production is greater in internal mammary arteries than in saphenous veins and is responsible for endothelium dependent relaxation seen in arteries in response to agents such as histamine, ${ }^{9}$ serotonin, ${ }^{18}$ and platelet derived $\mathrm{ADP}^{8}{ }^{8}$ In contrast, very small quantities of $\mathrm{NO}$ are released from veins in response to these agents, so they may cause little relaxation of veins or even constriction. Moreover, prostacyclin, which acts synergistically with $\mathrm{NO}$ as an inhibitor of platelet aggregation, is released in greater amounts by arteries than by veins. ${ }^{19}$ Thus, inhibition of platelet activation by endothelium in vein grafts is less than in arterial grafts and constriction rather than dilatation may be the response to platelet activation products such as thromboxane $A_{2}$ and serotonin. $^{8}$

In addition to local platelet activation within saphenous vein grafts, platelets may be activated systemically by cardiopulmonary bypass. Therefore, platelets perfusing the coronary grafts may already be activated and secreting granule contents. ${ }^{20}$ These granules release agents such as serotonin and ADP, causing constriction of the saphenous vein grafts, and platelet derived growth factors, which may promote subsequent proliferation of vascular smooth muscle cells. ${ }^{21}$ Although antiplatelet treatment with aspirin is effective in reducing vein graft occlusion rates, ${ }^{25}$ these are still higher than those of arterial grafts. Aspirin, however, is discontinued before surgery to prevent bleeding complications. Our results show that activated platelets sequestrate within the vein grafts before the end of the operation suggesting that effective antiplatelet treatment is required at this stage. The lack of NO production by vein grafts suggests that NO donors should be beneficial. Currently available NO donors, however, have only minor antiplatelet effects at therapeutic vasodilator concentrations ${ }^{10}$ and are thus limited in their usefulness as antiplatelet agents.

Recently, we reported that GSNO differs from other NO donors in that it has potent antiplatelet effects at doses that barely alter vasodilator tone in animals and humans. ${ }^{11} 1222$ In addition, GSNO prevents platelet activation during coronary angioplasty at doses that have no haemodynamic effect. ${ }^{13}$ Infusion of GSNO in our patients, at a dose that had no significant haemodynamic effect, decreased the number of activated platelets in vein and arterial grafts, the decrease in saphenous vein grafts being less than in internal mammary artery grafts in control patients. All patients in the in vivo study received glyceryl trinitrate. Although glyceryl trinitrate may exert a platelet inhibitory effect, ${ }^{23}$ this was not measured as its use is standard practice. Addition of GSNO, however, had a clear effect. Similarly, in vitro, the reduction in platelet count following passage of PRP through vein and artery segments was prevented by GSNO. This may have longer term advantages as preventing localisation of activated platelets within vein grafts, in addition to reducing early thrombosis, may reduce local release from platelets of factors such as platelet derived growth factor and transforming growth factors, which contribute to proliferation of vascular smooth muscle cell, an important factor in late vein graft occlusion. ${ }^{21}$

As saphenous vein grafts will, for technical reasons, continue to be needed in addition to arterial grafts, an antiplatelet agent is required at surgery. GSNO is effective in reducing platelet activation and localisation within saphenous vein grafts at a dose that has no haemodynamic effect. Therefore, GSNO or similar platelet specific NO donors may represent a useful treatment to improve vein graft patency. E J L was a Medical Research Council Training Fellow and J F
$M$ is British Heart Foundation Professor of Cardiovascular Science. We thank Dr Harold Hodson of Wellcome Research ence. We thank Dr Harold Hodson of Waboratories for the generous gift of GSNO.
Laborat

1 Chesebro JH, Clements IP, Fuster V, et al. A plateletinhibitor-drug trial in coronary-artery bypass operations. Benefit of perioperative dipyridamole and aspirin therapy on early postoperative vein-graft patency. $N$ Engl $7 \mathrm{Med}$ 1982;307:73-8.

2 RITA Trial Participants. Coronary angioplasty versus coronary artery bypass surgery: the randomised intervention treatment of angina (RITA) trial. Lancet 1993;341: tion treati 80.

3 Grondin CM, Campeau L, Lesperance J, et al. Comparison of late changes in internal mammary artery and saphenous vein grafts in two consecutive series of patients 10 years after operation. Circulation 1984;70(suppl 1):I208-12.

after operation. Circulation 1984;70(suppl 1):I208-12.
4 Bourassa MG. Long-term vein graft patency. Curr Opin Cardiol 1994;9:685-91.

5 Goldman S, Copeland J, Moritz T, et al. Saphenous vein graft patency 1 year after coronary artery bypass surgery and effects of antiplatelet therapy. Results of a veterans administration cooperative study. Circulation 1989;80: $1190-7$

6 Radomski MW, Palmer RMJ, Moncada S. Comparative pharmacology of endothelium-derived relaxing factor, nitric oxide and prostacyclin in platelets. Br f Pharmacol 1987;92:181-7.

7 Radomski MW, Palmer RMJ, Moncada S. Endogenous nitric oxide inhibits human platelet adhesion to vascular

8 Yang Z, Stultz P, von Segesser L, et al. Different interactions Yang $Z$, Stultz $P$, von Segesser L, et al. Different interactions
of platelets with arterial and venous coronary bypass vessels. Lancet 1991;337:939-43. 
9 Yang Z, von Segesser L, Bauer E, et al. Different activation of the endothelial L-arginine and cyclooxygenase pathway in the human internal mammary artery and saphenous vein. Circ Res 1991;68:52-60.

10 Mehta J, Mehta P. Comparative effects of nitroprusside and nitroglycerin on platelet aggregation in patients with hear failure. f Cardiovasc Pharmacol 1980;2:25-33.

11 de Belder AJ, MacAllister R, Radomski MW, et al. Effects of $\mathrm{S}$-nitrosoglutathione in the human forearm circulation: evidence for selective inhibition of platelet activation. Cardiovasc Res 1994;28:691-4.

12 Ramsey B, Radomski M, de Belder A, et al. Systemic effects of S-nitrosoglutathione in the human following intravenous infusion. Br f Clin Pharmacol 1995;40:101-2.

13 Langford EJ, Brown AS, Wainwright RJ, et al. Inhibition of platelet activity by S-nitrosoglutathione during coronary angioplasty. Lancet 1994;344:1458-60.

14 Miller DD, Rivera FJ, Garcia OJ, et al. Imaging of vascular injury with $99 \mathrm{mT}$ c-labelled monoclonal antiplatelet antibody S12. Preliminary experience in human percutaneous body S12. Preliminary experience in human percutane

15 Palabrica TM, Furie BC, Konstam MA, et al. Thrombus imaging in a primate model with antibodies specific for an external membrane protein of activated platelets. Proc Nat Acad Sci USA 1989;86:1036-41.

16 Mills SL, Denardo SJ, Denardo GL, et al. ${ }^{123}$ I radiolabelling of monoclonal antibodies for in vivo procedures. Hybri doma 1986;5:265-75.
17 Luscher TF, Diederich D, Siebenmann R, et al. Difference between endothelium-dependent relaxation in arterial and venous coronary bypass grafts. $N$ Engl F Med 1988;319: 462-7.

18 Yang Z, Diederich D, Schneider K, et al. Endotheliumderived relaxing factor and protection against contractions induced by histamine and serotonin in the human internal
mammary artery and in the saphenous vein. Circulation 1989;80:1041-8.

19 Subramanian VA, Hernandez Y, Tack-Goldman K, et al. Prostacyclin production by internal mammary artery as a factor in coronary artery bypass grafts. Surgery 1986;100: 376-83.

20 Rinder CS, Bohnert J, Rinder HM, et al. Platelet activation and aggregation during cardiopulmonary bypass. Anesthesiand aggregation during
ology 1991;75:388-93.

21 Bryan AJ, Angelini GD. The biology of saphenous vein graft occlusion: etiology and strategies for prevention. Curr Opin Cardiol 1994;9:641-9.

22 Radomski MW, Rees DD, Dutra A, et al. S-nitrosoglutathione inhibits platelet activation in vitro and in vivo. Br F Pharmacol 1992;107:745-9.

23 Langford EJ, Wainwright RJ, Martin JF. Platelet activation in acute myocardial infarction and unstable angina is inhibited by nitric oxide donors. Arterioscler Thromb Vasc Biol 1996;16:51-5. 